\title{
Addressing Older Latinos' Spiritual Needs in Hospital Settings: Identifying Predictors of Satisfaction
}

\author{
David R. Hodge \\ Robert J. Wolosin \\ Robin P. Bonifas
}

\begin{abstract}
Many older Latinos use spirituality to navigate the challenges associated with hospitalization. Although spiritual assessments are typically recommended in such settings, little, if any, research has sought to identify the factors that predict satisfaction with addressing clients' spiritual needs. Using a national sample of recently hospitalized older Latinos $(N=227)$, this secondary analysis identified predictors of older Latinos' satisfaction with how well their spiritual needs were addressed during their hospitalization. Among the eight predictors examined, room quality, nursing staff, and physicians were positively associated with satisfaction, while the discharge process was negatively associated with satisfaction. By understanding the factors that predict satisfaction, practitioners are better positioned to provide culturally relevant and effective services to older Latinos.
\end{abstract}

Keywords: Latinos, Hispanics, older adults, spirituality, health care settings

The population of older adults in the United States is diversifying and increasing rapidly. By 2050, the number of seniors is expected to double, and Latinos are projected to comprise approximately $20 \%$ of all people over age 60 (Administration on Aging, 2008a). In less than a decade, by 2019, Latinos are anticipated to be the largest cultural group represented among older adults (Administration on Aging, 2008b).

These demographic changes have important implications for social workers in health care settings. Many Latino elders will experience hospitalization and the attendant stress associated with admission to the healthcare system (Berkman, Silverstone, Simmons, Volland \& Howe, 2000). Spirituality, which is commonly understood as an individual's relationship with God (Wuthnow, 2007), is frequently used to navigate such situations (Soenke, Landau, \& Greenberg, 2013).

Furthermore, spirituality is often considered a basic Latino value (Koss-Chioino, 2013). To be clear, Latinos affirm a wide variety of spiritual beliefs and practices, which can vary by gender, culture of origin, and geographic location (Canda \& Furman, 2010). Thus, while the vast majority of Latinos are Catholic or evangelical Protestants (e.g., Pentecostal Christians), how these traditions are expressed can vary. This variation occurs at the micro level, between individual Latinos, as well as at the macro level, between Latinos collectively and European Americans. Indeed, based upon a nationally representative study, Pew Hispanic Center researchers concluded that Latinos practice a distinctive form of Christianity (Suro et al., 2007). Latin culture shapes how Christian

David R. Hodge, Ph.D., is an Associate Professor in the School of Social Work at Arizona State University in Phoenix and Senior Nonresident Fellow at the University of Pennsylvania's Program for Research on Religion and Urban Civil Society; Robert J. Wolosin, Ph.D, is affiliated with Press Ganey Associates, Inc., South Bend, IN; and Robin P. Bonifas, Ph.D., is an Assistant Professor in the School of Social Work at Arizona State University. This research was supported by a grant from the John A. Hartford Foundation.

Copyright (C) 2013 Advances in Social Work Vol. 14 No. 1 (Spring 2013), 65-81 
beliefs are interpreted and practiced (Koss-Chioino, 2013). The compartmentalization of spirituality, commonly found in European American culture, is largely absent. Rather, Latino spirituality is characterized by a spiritually-animated worldview that affirms the existence of a vibrant, present, non-material reality. This spiritually-informed worldview serves as a "moral compass" for navigating life (Suro et al., 2007).

Latino elders may rely upon this compass to guide them through the hospitalization experience, and social workers will need to be well prepared to effectively support them in this process. This paper presents the results of a study identifying factors associated with older Latinos' satisfaction with the attention given to their spiritual needs during hospitalization and provides social workers with foundational knowledge for delivering culturally relevant care. Before detailing study procedures and findings, an overview of spirituality in the context of healthcare, social work, and client or patient satisfaction is provided to orient the reader.

\section{Background}

Hospitalization frequently evokes spiritual needs, especially among devout populations such as older Latinos (Nelson-Becker, Nakashima, \& Canda, 2007). Spiritual needs are manifested in many different forms (Bussing, Balzar, \& Heusser, 2010; Cavendish et al., 2006; Hodge, Horvath, Larkin, \& Curl, 2012), but can be categorized under two basic rubrics: 1) assets that can be used to cope with or ameliorate challenges, and 2) barriers that can impede service provision (Hodge, 2005). The characteristics of each of these categories are summarized below, and examples are provided of specific needs in each category.

As an asset for coping, spirituality often plays an instrumental role in helping people deal with challenges (Pargament, 2007; Saleebey, 2009). A substantial and growing body of research indicates that spirituality is a significant source of strength that facilitates health, wellness, and resiliency in the face of adversity (Hook et al., 2010; Koenig, King, \& Carson, 2012; Koenig, McCullough, \& Larson, 2001; Pargament, 2007). For example, older Mexican Americans frequently turn to God to find the positive aspects of suffering, such as a sense of meaning or purpose in the experience (Krause \& Bastida, 2011).

To fully operationalize these spiritual assets in hospital settings, clients may have needs that must be addressed by healthcare personnel, and social workers are likely to be effectively positioned to begin a discussion of such needs. For example, previous qualitative research with non-Latino samples suggests that spiritual needs that facilitate coping are often manifested in two dimensions, vertical (i.e., needs related to connection with God), and horizontal (i.e., needs related to connecting with other human beings) (Conner \& Eller, 2004; Hermann, 2001). Regarding the former dimension, patients often report a need for prayer, meditation, Bible reading, listening to devotional music, and other activities perceived to facilitate a connection with God. Regarding the latter dimension, patients frequently report a need for interpersonal interactions, including interactions with hospital staff that are characterized by warmth, courteousness, caring, and reassurance. (Nixon \& Narayanasamy, 2010; Tanyi, Recine, Werner, \& Sperstad, 2006). This literature also suggests that hospital staff's efforts to provide patients a quiet 
room (conducive for prayer, connecting with God, etc.), attend to special requests (e.g., procure access to a Bible), facilitate visits by significant others, and demonstrate courtesy may help address patients' spiritual needs.

In terms of barriers to service provision, as implied above, spirituality often provides people with a worldview that informs them of who they are and how they should live (Koenig, 1998; Richards \& Bergin, 2000; Van Hook, Hugen, \& Aguilar, 2001). From a social work strengths perspective, diverse beliefs should not be viewed as a hindrance to care. Alternatively, it is important to recognize that service provision can conflict with clients' spiritual value systems.

For example, some Latinos may believe that aspects of curanderismo ${ }^{1}$ are at odds with certain western medical proscriptions such as precautions to avoid valued herbal remedies when taking western medications (Tafur, Crowe, \& Torres, 2009). Such conflicts can accentuate the stress associated with hospitalization and impede the healing process, requiring social workers to broker win-win solutions that respect Latino clients' spiritual values, while simultaneously maximizing adherence to essential aspects of care.

Prior qualitative research with non-Latino samples has illustrated a related set of spiritual needs (Davidson, Boyer, Casey, Matzel, \& Walden, 2008). Patients report a need to be active participants in treatment (Hodge \& Horvath, 2011; Meert, Thurston, \& Briller, 2005), and to have some degree of involvement and control during hospitalization (Hermann, 2001), which helps ensure that service provision is congruent with their spiritual beliefs and values. This literature also implies that hospital staff's efforts to keep patients informed and engaged, in tandem with staff's competency and responsiveness to requests may help address patients' spiritual needs.

The critical role that spiritual needs can play in service provision is implicitly recognized by the Joint Commission, the largest and most influential health care accrediting body in the United States. The Joint Commission revised its accreditation requirements at the turn of the century to necessitate spiritual assessments in hospitals and other settings (Hodge, 2006; Koenig, 2007). While each health care organization develops its own specific set of assessment procedures, the same overarching purpose exists across organizations: to identify patients' spiritual needs and determine the steps necessary to address the issues that emerge (Joint Commission, 2005). Put simply, the reason for conducting a spiritual assessment is to address clients' spiritual needs.

As might be expected, addressing patients' spiritual needs has been linked with patient satisfaction in studies using a cross-sectional research design. Patient satisfaction is an important construct because it is typically used to evaluate the quality of services provided in health care settings (Gribble \& Haupt, 2005; Press, 2002). Addressing patients' spiritual needs is associated with perceptions of global satisfaction with service provision. For instance, among a sample of patients receiving treatment from an outpatient cancer center in New York City $(N=369$; mean age $=58$ yrs, 65\% female; $67 \%$ white), those who reported their spiritual needs were not being adequately addressed

\footnotetext{
${ }^{1}$ Traditional healing methods common among many Latino cultural groups to address physical and spiritual maladies.
} 
also reported significantly lower levels of satisfaction with the medical care they received (Astrow, Wexler, Texeira, He, \& Sulmasy, 2007). Similarly, among hospital inpatients in Chicago $(N=3,141$; mean age $=56$ yrs, $61 \%$ female; $73 \%$ black $)$, those who reported discussing their spiritual concerns with a care provider reported higher levels of satisfaction with the care they received (Williams, Meltzer, Arora, Chung, \& Curlin, 2011). As such, addressing clients' spiritual needs appears to be an important component of overall satisfaction.

Despite the often crucial role that spiritual needs play in service provision, little empirical work has sought to examine predictors of clients' satisfaction with attention to their spirituality during hospitalization. As referred to above, some research has mapped the parameters of older adults' spiritual needs in healthcare settings (Cavendish et al., 2006; Hermann, 2001; Hodge et al., 2012; Murray, Kendall, Boyd, Worth, \& Benton, 2004; Tanyi et al., 2006). While research suggests that a range of spiritual needs may present, the factors associated with clients' satisfaction with spiritual care have yet to be identified.

This lack of knowledge represents an important gap in the literature, especially for older Latinos for whom spiritual needs may be particularly salient. Articulating factors that predict satisfaction can enable social workers and other healthcare professionals to effectively address clients' spiritual needs. Indeed, highlighting such variables can help practitioners tailor services toward this end. Accordingly, this study seeks to identify factors that predict older Latinos' satisfaction with how well their spiritual needs are addressed in hospital settings. The methods used to identify these predictors are delineated below.

\section{Method}

To identify predictors of satisfaction regarding older Latinos' spiritual needs, a secondary data analysis of hospital inpatient satisfaction data was conducted. The data was obtained from Press Ganey Associates, Inc., a healthcare consulting firm that specializes in patient satisfaction measurement and management. As referred to above, hospitals routinely assess patient satisfaction as a means to evaluate the quality of their services (Chandra et al., 2011). This task is frequently contracted out to other organizations, of which Press Ganey is the largest (Press Ganey Associates, 2011). Deidentified data was provided for the present study, which was conducted with the approval of a university Institutional Review Board.

\section{Participants}

The sample consisted of 227 Latinos ages 65 and older who were consecutively discharged over a 12-month period from hospitals across the nation. Some $56.8 \%(n=$ $129)$ of the sample was female. Just over three quarters $(76.7 \%, n=174)$ of respondents

were between 65 and 79 years old, while the remaining individuals were 80 and older. Regarding ethnicity, 36.1\% $(n=82)$ were Mexican American, $23.3 \%(n=53)$ were Puerto Rican, and the rest were "other Hispanic/Latino." Almost one third $(32.2 \% ; n=$ 
73) of respondents were from New England, 25.6\% $(n=58)$ were from the Midwest, and the remaining were from the South and Western regions of the nation.

\section{Survey Instrument}

To assess patients' perceptions, Press Ganey administers surveys to former inpatients soon after completion of the discharge process. Surveys are typically mailed out within five business days of receipt of the discharge data from client hospitals. Spanish language surveys are sent to patients whose language preference is noted in the demographic information provided by hospitals. The self-administered survey methodology tends to produce relatively accurate information about sensitive issues such as spirituality and the provision of medical care (Press, 2002).

The survey instrument included the demographic items reported above, several indicators of satisfaction, and the outcome variable: clients' assessment of how well their spiritual needs were addressed. The study included eight predictors that cover major areas of service provision during hospitalization. These eight areas also capture key dimensions of satisfaction delineated by the National Library of Health Care Indicators (Kaldenberg, 2001).

More specifically, respondents were asked to rate the quality of services they received in terms of the admission process, room quality, food service, nursing staff, the administration of tests and treatments, accommodation of visitors and family, physicians, and the discharge process. Prior qualitative research suggests that all of these areas could potentially be related to clients' spiritual needs (Cavendish et al., 2006; Hermann, 2001; Hodge et al., 2012; Koenig, 2012). Examples might include a quiet room in which to pray or meditate, food that conforms to religious proscriptions, and courteous interactions with hospital staff.

Each area was assessed with a multi-item scale; the shortest scale contained two items and the longest contained six items. For each item, respondents were presented with a 5-point, Likert-type response key that ranged from "very poor" (1) to "very good" (5). Table 1 lists the number of items in each scale, sample items, and each scale's Cronbach alpha. The reliability coefficients are consistent with alphas obtained in prior research (Kaldenberg, 2001), with values from .88 to .96, and suggest the scales performed well with this population (Spicer, 2005).

The outcome variable was assessed by asking respondents to indicate the "degree to which hospital staff addressed your spiritual needs" on the same 5-point, Likert-type response key described above. This item is similar to one used in prior quantitative work on spiritual needs in healthcare settings (Astrow et al., 2007). 
Table 1 Predictors, sample items, and reliability coefficients

\begin{tabular}{lcc}
\hline $\begin{array}{l}\text { Measure } \\
\text { (Sample item) }\end{array}$ & $\#$ & $\alpha$ \\
\hline $\begin{array}{l}\text { Admission process } \\
\text { (Courtesy of the person who admitted you) }\end{array}$ & 2 & .90 \\
$\begin{array}{l}\text { Quality of room } \\
\text { (Noise level in and around your room) }\end{array}$ & 5 & .89 \\
$\begin{array}{l}\text { Food service } \\
\text { (Courtesy of the person who served your food) }\end{array}$ & 3 & .88 \\
$\begin{array}{l}\text { Nursing staff } \\
\text { (Nurses' attitude toward your requests) }\end{array}$ & 6 & .96 \\
$\begin{array}{l}\text { Administration of tests and treatment } \\
\text { (Explanation about what would happen during tests or treatments) }\end{array}$ & 4 & .92 \\
$\begin{array}{l}\text { Visitors and family } \\
\text { (Accommodation and comfort for visitors) }\end{array}$ & 2 & .88 \\
$\begin{array}{l}\text { Physicians } \\
\text { (How well physician kept you informed) }\end{array}$ & 5 & .96 \\
$\begin{array}{l}\text { Discharge process } \\
\text { (Speed of the discharge process after you were told you could go home) }\end{array}$ & 3 & .88 \\
\hline
\end{tabular}

\section{Data Analysis}

Data were first screened for missing responses. Across the sample, $4.55 \%$ of values were missing. No data were missing from the outcome variable or the demographic variables. One of the items in the three-item admissions process scale was missing data from $17.2 \%(n=39)$ of cases. An additional eight items across four scales were missing data from $5.3 \%(n=12)$ to $9.7 \%(n=22)$ of cases. Missing data were imputed using the Expectation-Maximization algorithm procedure to enable statistical analysis of all cases (Schafer \& Graham, 2002).

Initially, bivariate relationships were examined between the satisfaction variables and the spiritual needs variable to determine general associations among the data. Statistical procedures included independent samples $t$-tests, one-way analysis of variance, and Pearson's correlation. Subsequently, hierarchical ordinary least squares (OLS) regression analyses were conducted to identify predictors of satisfaction associated with the degree to which staff addressed older Latino's spiritual needs. In block 1, demographic variables were included to examine the relationship between these variables and the outcome variable at the multivariate level. In block 2 , the eight satisfaction variables were added to 
identify significant predictors after controlling for the effects of the demographic variables. Variance inflation factors were well below 10, indicating little evidence of multicollinearity (Field, 2009).

\section{Results}

\section{Bivariate Analyses}

T-tests and one-way analysis of variance procedures were used to examine the relationships between the demographic variables and the spiritual needs variable. Gender, age, and Latino sub-group were not associated with clients' perceptions of how well their spiritual needs were met during their hospital stay. Conversely, geographic region was associated with satisfaction ( $p=.005)$, with Tukey's post hoc test indicating that older Latinos in the South and Western regions reported significant lower levels of satisfaction compared to respondents in the Midwest.

Pearson's correlation coefficients were computed to examine the relationships between the satisfaction variables and the spiritual needs variable. All eight predictors were associated with the outcome variable as expected. In other words, higher values on each of the predictors was associated with higher perceptions of satisfaction with the degree to which hospital staff addressed respondents' spiritual needs. Across the eight measures, the coefficients ranged from .52 (admissions process) to .70 (room quality); these values are commonly understood to indicate relatively strong associations (Cohen $\&$ Cohen, 1983).

\section{Multivariate Analyses}

To identify predictors of satisfaction after controlling for the effects of the demographic variables, a series of OLS regression analyses was conducted. As can be seen in Table 2, the first block consisted of the demographic variables. These variables accounted for approximately $4 \%$ of the variance in client satisfaction with how their spiritual needs were addressed. Consistent with the bivariate results, only one of the demographic variables was significant. Older Latinos in the Midwest reported higher levels of satisfaction with how staff addressed their spiritual needs when compared to the reference group, older Latinos in the South and West regions of the nation.

In block 2, the eight satisfaction variables were entered into the model as potential predictors. Results regarding the pattern of relationships among the demographic variables remained the same. As was the case with block 1, older Latinos in the Midwest region reported higher levels of satisfaction regarding their spiritual needs relative to the reference group. The inclusion of the predictors significantly increased the variance explained by the model, which accounted for approximately $57 \%$ of the variance in clients' satisfaction with how their spiritual needs were addressed. 
Table 2 Hierarchical regression analyses predicting satisfaction addressing spiritual needs

\begin{tabular}{|c|c|c|}
\hline & Block 1 & Block 2 \\
\hline \multicolumn{3}{|l|}{ Demographics } \\
\hline Female $^{1}$ & $-.200(.119)$ & $-.120(.080)$ \\
\hline Age $80+\mathrm{yrs}^{2}$ & $-.153(.139)$ & $-.030(.095)$ \\
\hline Puerto Rican ${ }^{3}$ & $.008(.160)$ & $.141(.109)$ \\
\hline Mexican American ${ }^{3}$ & $.022(.157)$ & $.028(.109)$ \\
\hline New England ${ }^{4}$ & $.303(.161)$ & $.126(.110)$ \\
\hline Midwest $^{4}$ & $.487(.155)^{* *}$ & $.358(.107)^{* *}$ \\
\hline \multicolumn{3}{|c|}{ Predicator of satisfaction addressing spiritual needs } \\
\hline Admission process & & $.010(.075)$ \\
\hline Quality of room & & $.462(.106)^{* * *}$ \\
\hline Food service & & $.134(.077)$ \\
\hline Nursing staff & & $1.831(.429)^{* * *}$ \\
\hline Administration of tests & & $.066(.121)$ \\
\hline Visitors and family & & $-.168(.089)$ \\
\hline Physicians & & $.238(.102)^{*}$ \\
\hline Discharge process & & $-.197(.087)^{*}$ \\
\hline Adjusted R square & $.037 *$ & $.566^{* * *}$ \\
\hline \multicolumn{3}{|c|}{$\begin{array}{l}\text { Unstandardized regression coefficients with standard errors in parentheses. } \\
\text { Reference groups: }{ }^{1} \text { male, }{ }^{2} \text { age } 65-79 \text { years, }{ }^{3} \text { other Latinos, }{ }^{4} \text { South and West regions. } \\
{ }^{*} p<.05, * * p<.01,{ }^{* * *} p<.001 .\end{array}$} \\
\hline
\end{tabular}

In terms of individual predictors, satisfaction with room quality, nursing staff, physicians, and the discharge process were significantly related to satisfaction with addressing spiritual needs after controlling for the effects of the demographic variables. The positive coefficients for room quality, nursing staff, and physicians indicate that higher levels of satisfaction with services in these areas predict higher levels of satisfaction with staff's efforts to address older Latinos' spiritual needs. Conversely, the negative coefficient for the discharge process indicates that higher levels of satisfaction with the discharge process predict lower levels of satisfaction with staff's efforts to address respondents' spiritual needs, holding all other values constant. 


\section{Discussion}

Hospital admission often engenders spiritual needs among older adults (NelsonBecker et al., 2007). In recognition of this reality, the Joint Commission requires an assessment of clients' spiritual needs to support the delivery of culturally-grounded care and services (Hodge, 2006; Koenig, 2007). Although addressing clients' spirituality is an important component of service provision (Astrow et al., 2007; Williams et al., 2011), surprisingly little is known about the implementation of this process (Koenig, 2007). For instance, to the best of our knowledge, little, if any, research has investigated factors that predict clients' satisfaction with the attention granted to their spiritual needs during hospitalization.

Given the growth of the older Latino population, the current study sought to address this gap in the literature by identifying factors that predict satisfaction with spiritual care in hospital settings. Before summarizing the results, it should be noted that the study employed a cross-sectional design. Thus, due to the correlational nature of the study, caution is warranted in interpreting the findings and their application. With this caveat in mind, the study identified a number of variables that were associated with satisfaction regarding efforts to address older Latinos' spiritual needs.

More specifically, key predictors identified include room quality and the quality of interactions with the nursing staff and physicians. In addition, the discharge process was negatively associated with satisfaction. The final model, which included the impact of all predictors, accounted for approximately $57 \%$ of the variance in older Latinos' satisfaction regarding the attention given their spiritual needs.

These results are consistent with prior qualitative research on spiritual needs (Cavendish et al., 2006; Hermann, 2001; Hodge et al., 2012). For example, past research with African Americans has highlighted the important role that God and nurses are perceived to play in spiritual care (Conner \& Eller, 2004; Tanyi et al., 2006). A room's noise level, its cleanliness, and temperature can have a direct bearing on clients' spiritual needs by, for example, inhibiting prayer and meditation and other practices that foster connection with God. Key members of the healthcare team such as nurses and doctors are also perceived to play a central role in addressing spiritual needs. They help respond to and fulfill requests, provide hope and interpersonal connection, and display warmth and empathy. Some older adults express a spiritual need to be kept informed about the state of their illness/progress, which was also a dimension tapped by the nurse and physician scales used in this study.

In addition to confirming prior research, the present study breaks new ground in at least three ways. First, it uses quantitative methods to document the relationship between the predictors and clients' spiritual needs previously suggested by qualitative work. Second, it extends the findings to older Latinos, whose spirituality tends to differ from the spirituality often expressed by European Americans (Suro et al., 2007). In turn, the specific knowledge obtained about this growing population can be used to promote greater cultural competency in service provision. Third, while much prior research on spiritual needs is based upon respondents at a single healthcare organization, the present study incorporated respondents from hospitals across the nation. This geographic 
diversity helps mitigate any extant regional biases and implies that the results are not an artifact of local dynamics.

\section{Implications}

These results have key implications for social work practice, education, and research. In the area of practice, the findings highlight the importance of personal interactions between healthcare providers and older Latinos. Hospital staff - nurses, physicians, social workers, and others-represent the vehicle through which spiritual needs can be addressed. To promote quality of care and patient satisfaction, staff must spend time actively engaging clients about issues related to their spirituality. At the same time, it is important to acknowledge that not all healthcare providers will feel comfortable discussing spiritual issues. An essential role for social workers in healthcare settings is to support multidisciplinary colleagues in gaining proficiency for conversing with clients about their spiritual needs.

Such training needs are especially salient given that, in spite of the Joint Commission's spiritual assessment requirements, research suggests that clients' spiritual needs are often overlooked in health care settings (Astrow et al., 2007; Balboni et al., 2007; Williams et al., 2011). For some older Latinos, this may not be problematic if they are uninterested in discussing their spiritual needs or if spiritual needs are not a salient personal issue. For others, however, the failure to identify and address spiritual needs can impede wellness (Cavendish et al., 2006; Davidson et al., 2008). Consequently, it is critical that older Latinos be afforded the opportunity to relate any extant spiritual needs or concerns. In turn, these concerns should be treated with dignity and respect by tailoring services appropriately; social workers can guide their multidisciplinary colleagues in the delivery of services that reflect and respect clients' spiritual values.

This same open posture towards clients' spiritual needs also applies to the discharge process. Interestingly, higher reported satisfaction with the discharge process was negatively associated with satisfaction addressing spiritual needs. Although more research is needed to confirm and further illuminate this relationship, the discharge process itself may elicit spiritual needs, especially in an era of financial constraints and attendant short hospitalizations. For example, older Latinos may not have worked through all the spiritual issues sparked by their admission to the healthcare system (Koenig, 2012). Thus, while satisfied with the speed of the discharge process, older Latinos may feel that the swiftness of their exit inhibits consideration of their spiritual needs. In these situations, social workers can enhance patient satisfaction and service quality by discussing strategies for post-discharge spiritual care by, for example, encouraging clients to connect with a cultural healer or religious leader if doing so is important to them.

Social workers can also play an important role facilitating connections and referrals. As implied earlier, older Latinos can express their spirituality in a variety of ways (Canda \& Furman, 2010). Expressions of spiritual need are likely to be similarly diverse (KossChioino, 2013). Maintaining a network of diverse contacts within the practitioner's 
hospital, healthcare community, and the broader local community can help address diverse spiritual needs.

The findings also underscore the need for further education and training on spiritual assessment. Most social workers, including most gerontological social workers, report receiving little training in the process of identifying and addressing clients' spiritual needs (Murdock, 2005; Sheridan, 2009). The paucity of training is problematic. Practitioners who have not been trained in the assessment process may feel ill-equipped to administer spiritual assessments in a professional and ethical manner that respects clients' spiritual autonomy (Canda \& Furman, 2010). In order to support multidisciplinary colleagues in addressing clients' spiritual needs, social workers will need to be firmly grounded in understanding and discussing such needs themselves.

Educational programs might acquaint future practitioners with various spiritual assessment tools (Canda \& Furman, 2010; Hodge, 2003; Koenig, 2007; Pargament \& Krumrei, 2009), along with their respective strengths and limitations (Hodge, 2005). Practitioners might also benefit from reading assessment approaches that operationalize the Joint Commission's assessment requirements (Hodge, 2006). Similarly, evidencedbased guidelines for the use of spiritual interventions may also be helpful to some practitioners (Hodge, 2011a; Hodge, 2011b).

Curricula might also include strengths-based content on the spiritual traditions commonly affirmed by Latinos such as Catholicism, evangelical Protestantism, and Pentecostalism (Suro et al., 2007). While it is important not to assume clients hold all tenets of a particular spiritual tradition, it is helpful to have some knowledge of beliefs and practices that are commonly held among Latinos. Such knowledge legitimates the topic of spirituality in healthcare settings, implicitly communicates respect for clients' traditions, and provides areas that can be tentatively explored with clients. Similarly, programs will want to include content that enables social work students to differentiate between incorporating clients' spiritual values into practice, which is within their scope of practice, and incorporating spiritual guidance into practice, which falls under the rubric of clergy (Hodge \& Bonifas, 2010).

Educators may benefit from visiting the Council on Social Work Education's (CSWE) Religion and Spirituality Clearinghouse website. The CSWE Clearinghouse features a number of peer-reviewed teaching modules and syllabi designed to promote ethical and effective practice with diverse expressions of spirituality. For example, among the resources included in the Religion and Spirituality Educational Resources section of the website is a syllabus on "Evidenced-based assessment and intervention."

The results also highlight the need for additional research. To better understand the discrete spiritual needs of older Latinos, in-depth interviews with patients might be conducted to delineate the nature and types of spiritual needs commonly manifested by Latinos from various spiritual traditions and cultures of origin. Research is also needed on the prevalence of spiritual assessments, the protocols used to operationalize assessments, the types of spiritually-oriented services available to patients, the training provided to staff, and other factors associated with ensuring patients' spiritual needs are adequately addressed. 
Given the paucity of research on predictors of satisfaction with attention to spiritual needs during hospitalization, the present study should be replicated with different samples of older Latinos, as well as general samples of Latinos. Research should also be conducted in other healthcare settings. Spiritual needs emerge in nursing homes and longterm care facilities and other settings served by social workers. Predictors of satisfaction regarding clients' spiritual needs may be different in diverse settings, indicating the need for additional research to identify areas of similarity and difference.

Research should also be conducted with African Americans, American Indians, and other racial/ethnic groups. Much like Latinos, these populations typically affirm distinct spiritual narratives (Richards \& Bergin, 2000; Van Hook et al., 2001). Accordingly, it is important that research be conducted that utilizes samples comprised solely or primarily of members of these groups. By identifying predictors of satisfaction, services can be tailored to enhance their cultural relevance and effectiveness.

\section{Limitations}

As noted at the start of the discussion section, the cross-sectional design precludes any assessment of causality. In keeping with the correlated nature of the data, other models may also be plausible. Satisfaction with efforts to address patients' spiritual needs may be more accurately predicted by respondents' physical health status, religious affiliation, self-ascribed importance attributed to spirituality, or some other variable or combination of variables not included in the present study.

Although the study incorporated respondents from diverse regions of the nation, the findings should not be generalized to all hospitalized older Latinos in the United States; hence, the need for replication with other samples of older Latinos. It should also be noted that social desirability bias can affect survey responses (Williams et al., 2011). Finally, single-item measures such as the outcome variable employed in this study are characterized by limited reliability and validity. Concurrently, it should also be mentioned that such measures have been shown to function reasonably well in previous research despite their psychometric limitations (Bond, Dickinson, Matthews, Jagger \& Brayne, 2006; Menec, Shooshtari \& Lambert, 2007; Zimmerman et al., 2006), suggesting some degree of confidence in the findings is warranted.

\section{Conclusion}

To the best of our knowledge, this is the first study to identify predictors of older Latinos' satisfaction with how well their spiritual needs were addressed during hospitalization. In light of the rapidly growing population of older Latinos (Administration on Aging, 2008b) and the critical role that spirituality plays among this population (Suro et al., 2007), this study makes an important contribution to the literature. The findings suggest that room quality, nursing staff, physicians, and the discharge process play an important role-either positively or negatively-in satisfactorily addressing older Latinos' spiritual needs. By understanding the role such predictors play in clients' experiences, social work practitioners and their 
multidisciplinary colleagues are better equipped to provide culturally relevant and effective services to older Latinos.

\section{References}

Administration on Aging. (2008a). Population projections by race and Hispanic origin for persons 60 and over: 2000 to 2050. Retrieved 06/05/2010, from http://www.aoa.gov/AoARoot/Aging_Statistics/future_growth/future_growth.aspx.

Administration on Aging. (2008b). A statistical profile of Hispanic older Americans aged $65+$. Retrieved from http://www.aoa.gov/AoARoot/Aging_Statistics/Minority_Aging/Facts-on-HispanicElderly.aspx

Astrow, A. B., Wexler, A., Texeira, K., He, M. K., \& Sulmasy, D. P. (2007). Is failure to meet spiritual needs associated with cancer patients' perceptions of quality of care and their satisfaction with care? Journal of Clinical Oncology, 25(36), 5753-5757.

Balboni, T. A., Vanderwerker, L. C., Block, S. D., Paulk, M. E. L., Christopher S., Peteet, J. R., \& Prigerson, H. G. (2007). Religiousness and spiritual support among advanced cancer patients and associations with end-of-life treatment preferences and quality of life. Journal of Clinical Oncology, 25(5), 555-560.

Berkman, B. J., Silverstone, B., Simmons, W. J., Volland, P. J., \& Howe, J. L. (2000). Social work gerontological practice: The need for faculty development in the new millennium. Journal of Gerontological Social Work, 34(1), 5-23.

Bond, J., Dickinson, H. O., Matthews, F., Jagger, C., \& Brayne, C. (2006). Self-rated health status as a predictor of death, functional and cognitive impairment: A longitudinal cohort study. European Journal of Aging, 3, 193-206.

Bussing, A., Balzar, H. J., \& Heusser, P. (2010). Spiritual needs of patients with chronic pain diseases and cancer: Validation of the spiritual needs questionnaire. European Journal of Medical Research, 15(6), 266-273.

Canda, E. R., \& Furman, L. D. (2010). Spiritual diversity in social work practice: The heart of helping ( $2^{\text {nd }}$ ed.). New York: Oxford University Press.

Cavendish, R., Como, J., Konecny, L., Lanza, M., Luise, B. K., Mitzeliotis, C., ... Okumakpeyi, P. (2006). Patients' perceptions of spirituality and the nurse as a spiritual care provider. Holistic Nursing Practice, 20(1), 41-47.

Chandra, A., Sieck, S., Hocker, M., Gerardo, C. J., Villani, J., Harrison, D., ... Limkakneg, A. (2011). An observation unit may help improve an institution's Press Ganey satisfaction score. Critical Pathways in Cardiology, 10(2), 104-106.

Cohen, J., \& Cohen, P. (1983). Applied multiple regression/correlation analysis for the behavioral sciences ( $2^{\text {nd }}$ ed.). Hillsdale, NJ: Lawrence Erlbaum. 
Conner, N. E., \& Eller, L. S. (2004). Spiritual perspectives, needs and nursing interventions of Christian African Americans. Journal of Advanced Nursing, 46(6), 624-632.

Davidson, J. E., Boyer, M. L., Casey, D., Matzel, S. C., \& Walden, D. (2008). Gap analysis of cultural and religious needs of hospitalized patients. Critical Care Nursing Quarterly, 31(2), 119-126.

Field, A. (2009). Discovering statistics using SPSS (3rd ed.). Thousand Oaks, CA: Sage.

Gribble, R. K., \& Haupt, C. (2005). Quantitative and qualitative differences between handout and mailed patient satisfaction surveys. Medical Care, 43(3), 276-281.

Hermann, C. P. (2001). Spiritual needs of dying patients: A qualitative study. Ontology Nursing Forum, 28(1), 67-72.

Hodge, D. R. (2003). Spiritual assessment: A handbook for helping professionals. Botsford, CT: NACSW.

Hodge, D. R. (2005). Developing a spiritual assessment toolbox: A discussion of the strengths and limitations of five different assessment methods. Health and Social Work, 30(4), 314-323.

Hodge, D. R. (2006). A template for spiritual assessment: A review of the JCAHO requirements and guidelines for implementation. Social Work, 51(4), 317-326.

Hodge, D. R. (2011a). Evidenced-based spiritual practice: Using research to inform the selection of spiritual interventions. Journal of Religion and Spirituality in Social Work: Social Thought, 30(4), 325-339.

Hodge, D. R. (2011b). Using spiritual interventions in practice: Developing some guidelines from evidenced-based practice. Social Work, 56(2), 149-158.

Hodge, D. R., \& Bonifas, R. P. (2010). Using spiritually modified CBT to help clients wrestling with depression: A promising intervention for some older adults. Journal of Religion and Spirituality in Social Work: Social Thought, 29(3), 185-206.

Hodge, D. R., \& Horvath, V. E. (2011). Spiritual needs in health care settings: A qualitative meta-synthesis of clients' perspectives. Social Work, 56(4), 306-316.

Hodge, D. R., Horvath, V. E., Larkin, H., \& Curl, A. L. (2012). Older adults' spiritual needs in healthcare settings: A qualitative meta-synthesis. Research on Aging, 34(2), 131-155.

Hook, J. N., Worthington, E. L., Davis, D. E., Jennings, D. J., Gartner, A. L., \& Hook, J. P. (2010). Empirically supported religious and spiritual therapies. Journal of Clinical Psychology, 66(1), 46-72.

Joint Commission. (2005). Asked and answered: Evaluating your spiritual assessment process. The Source, 3(2), 6-7.

Kaldenberg, D. O. (2001). Patient satisfaction and health status. Health Marketing Quarterly, 18(3/4), 81-101. 
Koenig, H. G. (Ed.). (1998). Handbook of religion and mental health. New York, NY: Academic Press.

Koenig, H. G. (2007). Spirituality in patient care ( $2^{\text {nd }}$ ed.). Philadelphia, PA: Templeton Foundation Press.

Koenig, H. G. (2012). Religion, spirituality, and health: The research and clinical implications. ISRN Psychiatry, 2012(Art. ID 278730), 33 pages. doi: $10.5402 / 2012 / 278730$

Koenig, H. G., King, D., \& Carson, V. B. (2012). Handbook of religion and health (2nd ed.). New York, NY: Oxford University Press.

Koenig, H. G., McCullough, M. E., \& Larson, D. B. (2001). Handbook of religion and health. New York, NY: Oxford University Press.

Koss-Chioino, J. (2013). Religion and spirituality in Latino life in the United States. In K. I. Pargament (Ed.), APA handbook of psychology, religion, and spirituality: Vol. 1. Context, theory, and research (pp. 599-615). Washington, DC: American Psychological Association.

Krause, N., \& Bastida, E. (2011). Religion, suffering, and self-rated health among older Mexican Americans. Journal of Gerontology: Social Sciences, 66B(2), 207-216. doi: $10.1093 /$ geronb/gbq086

Meert, K. L., Thurston, C. S., \& Briller, S. H. (2005). The spiritual needs of parents at the time of their child's death in the pediatric intensive care unit and during bereavement: A qualitative study. Pediatric Critical Care Medicine, 6(4), 420-427.

Menec, V. H., Shooshtari, S., \& Lambert, P. (2007). Ethnic differences in self-rated health among older adults. Journal of Aging and Health, 19(1), 62-86.

Murdock, V. (2005). Guided by ethics: Religion and spirituality in gerontological social work practice. Journal of Gerontological Social Work, 45(1/2), 131-154.

Murray, S. A., Kendall, M., Boyd, K., Worth, A., \& Benton, T. F. (2004). Exploring the spiritual needs of people dying of lung cancer or heart failure: A prospective qualitative interview study of patients and their careers. Palliative Medicine, 18(1), 39-45.

Nelson-Becker, H., Nakashima, M., \& Canda, E. R. (2007). Spiritual assessment in aging: A framework for clinicians. Journal of Gerontological Social Work, 48(3/4), 331-347.

Nixon, A., \& Narayanasamy, A. (2010). The spiritual needs of neuro-oncology patients from patients' perspective. Journal of Clinical Nursing, 19, 2259-2270.

Pargament, K. I. (2007). Spiritually integrated psychotherapy: Understanding and addressing the sacred. New York, NY: Guilford Press.

Pargament, K. I., \& Krumrei, E. J. (2009). Clinical assessment of clients' spirituality. In J. D. Aten \& M. M. Leach (Eds.), Spirituality and the therapeutic process: A 
comprehensive resource from intake to termination (pp. 93-120). Washington, DC: American Psychological Association.

Press Ganey Associates. (2011). Hospital pulse report: Patient perspectives on American health care. South Bend, IN.

Press, I. (2002). Patient satisfaction: Defining, measuring, and improving the experience of care. Chicago, IL: Health Administration Press.

Richards, P. S., \& Bergin, A. E. (Eds). (2000). Handbook of psychotherapy and religious diversity. Washington, DC: American Psychological Association.

Saleebey, D. (Ed.). (2009). The strengths perspective in social work practice ( $5^{\text {th }} \mathrm{ed}$.). Boston: Pearson/Allyn \& Bacon.

Schafer, J. L., \& Graham, J. W. (2002). Missing data: Our view of the state of the art. Psychological Methods, 7(2), 147-177.

Sheridan, M. (2009). Ethical issues in the use of spiritually based interventions in social work practice: What we are doing and why. Journal of Religion and Spirituality in Social Work, 28(1/2), 99-126.

Soenke, M., Landau, M. J., \& Greenberg, J. (2013). Sacred armor: Religion's role as a buffer against the anxieties of life and the fear of death. In K. I. Pargament (Ed.), APA handbook of psychology, religion, and spirituality: Vol. 1. Context, theory, and research (pp. 105-122). Washington, DC: American Psychological Association.

Spicer, J. (2005). Making sense of multivariate data analysis. Thousand Oaks, CA: Sage Publications.

Suro, R., Escobar, G., Livingston, G., Hakimzadeh, S., Lugo, L., Stencel, S., ... Chaudhry, S. (2007). Changing faiths: Latinos and the transformation of American religion. Retrieved from http://www.pewforum.org/uploadedfiles/Topics/Demographics/hispanics-religion07-final-mar08.pdf

Tafur, M. M., Crowe, T. K., \& Torres, E. (2009). A review of curanderismo and healing practices among Mexicans and Mexican Americans. Occupational Therapy International, 16(1), 82-88.

Tanyi, R. A., Recine, A. C. G., Werner, J. S., \& Sperstad, R. A. (2006). Perceptions of incorporating spirituality into their care: A phenomenological study of female patients on hemodialysis. Nephrology Nursing Journal, 33(5), 532-538.

Van Hook, M., Hugen, B., \& Aguilar, M. A. (Eds). (2001). Spirituality within religious traditions in social work practice. Pacific Grove, CA: Brooks/Cole.

Williams, J. A., Meltzer, D., Arora, V., Chung, G., \& Curlin, F. A. (2011). Attention to inpatients' religious and spiritual concerns: Predictors of association with patient satisfaction. Journal of General Internal Medicine, 26(11), 1265-1271. 
Wuthnow, R. (2007). After the Baby Boomers: How twenty- and thirty-somethings are shaping the future of American religion. Princeton, NJ: Princeton University Press.

Zimmerman, M., Ruggero, C. J., Chelminski, I., Young, D., Posternak, M. A., Friedman, M., ... Attiullah, N. (2006). Developing brief scales for use in clinical practice: The reliability and validity of single-item self-report measures of depression symptom severity, psychosocial impairment due to depression, and quality of life. Journal of Clinical Psychiatry, 67(10), 1536-1541.

\section{Author note:}

Address correspondence to: David R. Hodge, Ph.D., School of Social Work, Mail Code 3920, 411 N. Central, Suite 800, Phoenix, Arizona, 85004-0689. 